\title{
Trichoderma na promoção do crescimento vegetal
}

\section{Lillian França Borges Chagas ${ }^{1}$, Aloisio Freitas Chagas Junior ${ }^{1}$, Layssah Passos Soares ${ }^{1}$, Rodrigo Ribeiro Fidelis ${ }^{1}$}

${ }^{1}$ Universidade Federal do Tocantins, Campus de Gurupi, Gurupi, Tocantins, Brasil. E-mail: lillianfbc@uft.edu.br, chagasjraf@uft.edu.br, layssahsoares@hotmail.com, fidelisrr@mail.uft.edu.br

Recebido: 23/02/2017; Aceito: 17/07/2017.

\section{RESUMO}

Fungos do gênero Trichoderma são uns dos principais micro-organismos de importância para o aumento do crescimento vegetal. Desta forma, objetivou-se com este trabalho avaliar a eficiência da inoculação de Trichoderma asperellum UFT 201, como promotores de crescimento vegetal nas culturas da soja, feijão caupi, arroz e milho, em casa de vegetação. As características de biomassa das culturas foram determinadas através da massa seca da parte aérea, massa seca da raiz e massa seca total, bem como a eficiência relativa. Nas diferentes culturas estudadas, a inoculação de T. asperellum UFT 201 foi superior para as características de biomassa, mostrando o potencial como promotor de crescimento, com aumento acima de $60 \%$ em relação à testemunha, para todas as culturas. Os resultados demonstram a capacidade do inoculante Trichoderma asperellum UFT 201, em promover o crescimento inicial de plantas, como observado para as culturas estudadas.

Palavras-chave: biomassa, culturas agrícolas, bioinoculante

\section{Trichoderma in promoters of plant growth}

\begin{abstract}
Fungi of the genus Trichoderma are one of the major importance of microorganisms to increase plant growth. Thus, the aim of this work was to evaluate the efficiency of Trichoderma asperellum UFT 201 inoculation, as plant growth promoters in the soybean, cowpea, rice and corn, in the greenhouse. The biomass crop characteristics were determined by dry weight of shoot, root dry mass and total dry mass and the relative efficiency. In the different plant species studied, the inoculation was higher for T. asperellum UFT 201 biomass characteristics, showing its potential as a growth promoter, an increase higher than $60 \%$ compared to control for all cultures. The results demonstrate the ability of $T$. asperellum UFT 201 inoculant, to promote the initial growth of plants, as observed for the studied cultures.
\end{abstract}

Key words: biomass, crops, bioinoculant. 
A influência de micro-organismos sobre o desenvolvimento das plantas é ampla, incluindo os efeitos benéficos na germinação de sementes, emergência de plântulas, crescimento e produtividade de grãos. A utilização de promotores de crescimento de plantas para o aumento da produção agrícola será provavelmente uma das táticas mais importantes para a atualidade no mundo. Isso se deve à demanda emergente para a diminuição da dependência de fertilizantes minerais e da necessidade para o desenvolvimento de uma agricultura sustentável (MACHADO et al., 2012).

A produção de inoculantes de baixo custo com micro-organismos promotores de crescimento de plantas é uma alternativa para diminuir os riscos ambientais causados pela utilização inadequada, e às vezes excessiva, de insumos e agrotóxicos. Os promotores de crescimento das plantas também contribuem para aumentar a produção agrícola, tornar o produto mais competitivo e diferenciado e, ainda, diminuir os custos para o produtor (POMELLA; RIBEIRO, 2009; MACHADO et al., 2012).

Fungos do gênero Trichoderma são uns dos principais micro-organismos de importância para o aumento do crescimento vegetal. Este fungo pode influenciar positivamente na germinação de sementes, no desenvolvimento e rendimento da cultura devido, também, à produção de substâncias promotoras de crescimento e melhoria na nutrição das plantas, principalmente pela solubilização de fósforo (OLIVEIRA et al., 2012; SILVA et al., 2012) e síntese de ácido indol acético (OLIVEIRA et al., 2012; CHAGAS et al., 2016), tendo grande importância econômica para a agricultura, também, por serem capazes de atuarem como agentes de controle de doenças de várias plantas cultivadas e indutores de resistência de doenças nas plantas (CONTRERASCORNEJO et al., 2009; SANTOS et al., 2012; SILVA et al., 2012; ASUMING-BREMPONG, 2013).

Objetivou-se com este trabalho avaliar a eficiência da inoculação de Trichoderma, como promotores de crescimento vegetal na cultura da soja [Glycine max (L.) Merrill.], feijão caupi [Vigna unguiculata (L.) Walp.], arroz (Oryza sativa L.) e milho (Zea mays L.), em casa de vegetação.

Foram utilizadas duas espécies de Trichoderma, o $T$. asperellum UFT 201 e T. harzianum, identificados através do sequenciamento da região ITS (Internal Transcribed Spacer), no Instituto Biológico, São Paulo, cujo número de acesso no GenBank e referências dos autores que depositaram o sequenciamento das espécies identificadas, encontram-se apresentadas na tabela 1 .

Para o preparo dos inoculantes a base de Trichoderma, os isolados foram colocados para crescer separadamente em placa de petri contendo meio BDA (batata dextrose ágar - Himedia, Índia) e incubados a temperatura de $25{ }^{\circ} \mathrm{C} \pm 2{ }^{\circ} \mathrm{C}$ com fotoperíodo de 12 horas, por sete dias, período determinado para o crescimento das colônias de Trichoderma spp.

Tabela 1. Espécies de Trichoderma identificadas através do sequenciamento da região ITS, utilizados nos experimentos.

\begin{tabular}{lccc}
\hline $\begin{array}{l}\text { Espécies de } \\
\text { Trichoderma }\end{array}$ & Acesso & IS & Referência \\
\hline $\begin{array}{l}\text { T. harzianum CIB } \\
\text { T23 }\end{array}$ & EU279989 & 100 & Hoyos-Carvajal et \\
$\begin{array}{l}\text { T. asperellum GJS } \\
\text { 04-217 }\end{array}$ & DQ381958 & 100 & $\begin{array}{c}\text { al. (2009) } \\
\text { Samuels et al. } \\
(2010)\end{array}$ \\
\hline
\end{tabular}

IS: Índice de similaridade.

Sacos de polipropileno contendo $300 \mathrm{~g}$ do arroz e $300 \mathrm{~mL}$ de água destilada foram autoclavados a $121{ }^{\circ} \mathrm{C}$, por 1 hora. Após o resfriamento, o arroz foi inoculado com seis discos de $5 \mathrm{~mm}$ de diâmetro de cada isolado, separadamente, contendo micélios e esporos de Trichoderma spp. e meio BDA e incubados em câmera de crescimento tipo B.O.D. com temperatura de $25^{\circ} \mathrm{C} \pm$ $2{ }^{\circ} \mathrm{C}$ e fotoperíodo de 12 horas por sete dias. A cada dois dias, o substrato contendo arroz foi revolvido, para facilitar a troca gasosa, quebra dos agregados miceliais e aumento da esporulação. Após os sete dias de incubação, o arroz foi peneirado em peneira de malha de $2 \mathrm{~mm}$ para separação dos esporos. Em seguida os esporos foram misturados em caulim como veículo para preparo do inoculante em pó.

A concentração de Trichoderma dos isolados utilizados foi determinada pelo método de diluição em série através da quantificação de Unidades Formadoras de Colônias (UFC), onde foi determinado utilizando-se $1 \mathrm{~g}$ de caulim em $10 \mathrm{~mL}$ de água esterilizada, seguida de agitação por 1 min., em seguida feitas as diluições e plaqueamento em placa de petri contendo meio BDA. Foi utilizada no experimento em média concentrações de $2 \times 10^{8}$ UFC por grama de caulim.

Os experimentos em casa de vegetação foram conduzidos na estação experimental da Universidade Federal do Tocantins, Campus Universitário de Gurupi, localizado na região sul do Estado do Tocantins (11 43'45" S, 4904'07" W, 280 m altitude). Para a instalação dos experimentos foram utilizados vasos com capacidade de $2,0 \mathrm{~kg}$, preenchidos com solo classificado como Latossolo Vermelho Amarelo distrófico (peneirado) de textura média, na profundidade de 0-20 $\mathrm{cm}$, obtido na própria estação Experimental da UFT, onde foram encontradas as seguintes características: 4,0 cmolc $\mathrm{dm}^{-3}$ de $\mathrm{Ca} ; 0,9 \mathrm{cmolc} \mathrm{dm}^{-3}$ de $\mathrm{Mg} ; 0,1 \mathrm{cmolc} \mathrm{dm}^{-}$ ${ }^{3}$ de $\mathrm{K} ; 2,8 \mathrm{mg} \mathrm{dm}^{-3}$ de P; 0,06 cmolc $\mathrm{dm}^{-3}$ de $\mathrm{Al} ; 8,3$ cmolc $\mathrm{dm}^{-3}$ de CTC; 5,0 cmolc $\mathrm{dm}^{-3}$ de soma de bases (S); $61 \%$ de saturação de bases (V); pH 5,8 em água; $1,7 \%$ de matéria orgânica; textura de 79, 5,0 e 16\% de 
areia, silte e argila, respectivamente: $\mathrm{P}$ e $\mathrm{K}$ - extrator Mehich 1; $\mathrm{Al}^{3+}, \mathrm{Ca}^{2+}$ e $\mathrm{Mg}^{2+}$ - Extrator $\mathrm{KCl}\left(1 \mathrm{~mol} \mathrm{~L}^{-1}\right)$ (EMBRAPA, 2009). Foi realizada adubação de base (N$\mathrm{P}-\mathrm{K}+$ micronutrientes) no solo antes do plantio conforme análise de solo e recomendações para cada cultura utilizando-se superfosfato simples, cloreto de potássio, ureia e FTE.

Foram semeadas oito sementes por vaso para cada cultura sendo feito o desbaste cinco dias após a germinação deixando uma planta por vaso para as culturas do feijão caupi, soja e milho e duas plantas por vaso para a cultura do arroz. As sementes de feijão caupi e soja foram inoculadas com rizóbio (Bradyrhizobium sp.). No feijão caupi utilizou-se a estirpe SEMIA 6462, e em soja as estirpes SEMIA 5079 e SEMIA $5080\left(3 \times 10^{9} \mathrm{UFC} \mathrm{mL}^{-1}\right)$. A inoculação com rizóbio aconteceua uma hora antes do plantio na concentração de $500 \mathrm{~mL}$ para $50 \mathrm{~kg}^{-1}$ de sementes.

Os tratamentos utilizados foram inoculação de $\mathrm{T}$. asperellum UFT 201, inoculação do fungo padrão Trichoderma harzianum (Controle) e uma testemunha sem inoculação de produto biológico (Controle absoluto). Utilizou-se como controle positivo o inoculante a base de T. harzianum por ser a cepa mais comercializada no Brasil e em vários países como princípio ativo de inoculantes de efeito biofungicida e promotor de crescimento de plantas. Os inoculantes foram aplicados diretamente nas sementes uma hora antes do plantio com dose de $20 \mathrm{~g}$ por $\mathrm{kg}$ de sementes, concentração recomendada para inoculação de sementes com as concentrações de Trichoderma em pó.

$\mathrm{O}$ delineamento experimental foi inteiramente ao acaso com três tratamentos e oito repetições, sendo quatro repetições para cada avaliação, em experimentos independentes para cada cultura. Para a determinação de biomassa foram feitas duas avaliações: a primeira avaliação aos 10 dias após emergência (DAE) para a cultura do milho e aos 20 DAE para arroz, soja e feijão caupi. A segunda realizada aos 25 DAE para as culturas do milho, e aos 45 DAE para arroz, soja e feijão caupi. Para a cultura do milho as avaliações aos 10 e 20 DAE foram em função do crescimento acelerado nos vasos. $\mathrm{O}$ material coletado foi lavado em água corrente e levado para secar em estufa a $60{ }^{\circ} \mathrm{C}$ para determinação da massa seca da parte aérea (MSPA), massa seca da raiz (MSR) e massa seca total (MST). Com os dados de MSPA determinou-se a eficiência relativa de cada tratamento calculada segundo a fórmula: $\mathrm{ER}=(\mathrm{MSPA}$ inoculada com Trichoderma / MSPA sem inoculante) $\mathrm{x}$ 100 .

Para os efeitos da inoculação em milho, na primeira avaliação aos 10 dias após a emergência (DAE), não houve diferença significativa entre os tratamentos (Tabela 2).

Tabela 2. Massa seca da parte aérea (MSPA), massa seca da raiz (MSR) e massa seca total (MST), em milho, arroz, soja e feijão caupi inoculados com Trichoderma, em casa de vegetação ${ }^{1}$.

\begin{tabular}{|c|c|c|c|c|c|c|}
\hline Tratamentos & $\begin{array}{c}\text { MSPA } \\
(\mathrm{g})\end{array}$ & $\begin{array}{c}\text { MSR } \\
(\mathrm{g})\end{array}$ & $\begin{array}{c}\text { MST } \\
(\mathrm{g}) \\
\end{array}$ & $\begin{array}{c}\text { MSPA } \\
(\mathrm{g})\end{array}$ & $\begin{array}{c}\text { MSR } \\
(\mathrm{g})\end{array}$ & $\begin{array}{c}\text { MST } \\
(\mathrm{g})\end{array}$ \\
\hline Milho & \multicolumn{3}{|c|}{$10 \mathrm{DAE}^{(2)}$} & \multicolumn{3}{|c|}{$25 \mathrm{DAE}$} \\
\hline T. asperellum UFT 201 & $0,54 \mathrm{a}$ & $1,61 \mathrm{a}$ & $2,15 \mathrm{a}$ & $7,64 \mathrm{a}$ & $3,74 \mathrm{a}$ & $11,38 \mathrm{a}$ \\
\hline T. harzianum & $0,49 \mathrm{a}$ & $1,55 \mathrm{a}$ & $2,04 \mathrm{a}$ & $5,66 \mathrm{~b}$ & $2,55 \mathrm{~b}$ & $8,21 \mathrm{~b}$ \\
\hline Testemunha & $0,47 \mathrm{a}$ & $1,45 \mathrm{a}$ & $1,92 \mathrm{a}$ & $4,29 \mathrm{c}$ & $2,82 \mathrm{c}$ & $7,11 \mathrm{c}$ \\
\hline $\mathrm{CV}(\%)^{3}$ & 14,5 & 18,5 & 14,8 & 11,2 & 13,3 & 9,8 \\
\hline Arroz & \multicolumn{3}{|c|}{$20 \mathrm{DAE}$} & \multicolumn{3}{|c|}{$45 \mathrm{DAE}$} \\
\hline T. asperellum UFT 201 & $0,45 \mathrm{a}$ & $0,55 \mathrm{a}$ & 0,99 a & $2,17 \mathrm{a}$ & $2,84 \mathrm{a}$ & $5,01 \mathrm{a}$ \\
\hline T. harzianum & $0,27 \mathrm{~b}$ & $0,30 \mathrm{~b}$ & $0,57 \mathrm{~b}$ & $1,53 \mathrm{~b}$ & $1,06 \mathrm{~b}$ & $2,59 \mathrm{~b}$ \\
\hline Testemunha & $0,21 \mathrm{~b}$ & $0,21 \mathrm{~b}$ & $0,42 \mathrm{~b}$ & $1,11 \mathrm{~b}$ & $1,15 \mathrm{~b}$ & $2,25 \mathrm{~b}$ \\
\hline $\mathrm{CV}(\%)$ & 20,6 & 29,7 & 16,5 & 21,1 & 22,9 & 18,6 \\
\hline Soja & \multicolumn{3}{|c|}{$20 \mathrm{DAE}$} & \multicolumn{3}{|c|}{$45 \mathrm{DAE}$} \\
\hline T. asperellum UFT 201 & $0,90 \mathrm{a}$ & $0,69 \mathrm{a}$ & $1,59 \mathrm{a}$ & $2,17 \mathrm{a}$ & $1,49 \mathrm{a}$ & $3,66 \mathrm{a}$ \\
\hline T. harzianum & $0,88 \mathrm{a}$ & $0,59 \mathrm{a}$ & $1,47 \mathrm{a}$ & $1,44 \mathrm{~b}$ & $1,05 \mathrm{~b}$ & $2,49 \mathrm{~b}$ \\
\hline Testemunha & $0,54 \mathrm{~b}$ & $0,35 \mathrm{~b}$ & $0,89 \mathrm{~b}$ & $0,95 \mathrm{c}$ & $0,86 \mathrm{c}$ & $1,81 \mathrm{c}$ \\
\hline $\mathrm{CV}(\%)$ & 23,2 & 30,4 & 20,8 & 28,3 & 18,9 & 14,6 \\
\hline Feijão caupi & \multicolumn{3}{|c|}{$20 \mathrm{DAE}$} & \multicolumn{3}{|c|}{$45 \mathrm{DAE}$} \\
\hline T. asperellum UFT 201 & $1,09 \mathrm{a}$ & $0,74 \mathrm{a}$ & $1,84 \mathrm{a}$ & $2,31 \mathrm{a}$ & $1,57 \mathrm{a}$ & $3,88 \mathrm{a}$ \\
\hline T. harzianum & $1,11 \mathrm{a}$ & $0,73 \mathrm{a}$ & $1,84 \mathrm{a}$ & $1,44 \mathrm{~b}$ & $1,05 \mathrm{~b}$ & $2,49 \mathrm{~b}$ \\
\hline Testemunha & $0,57 \mathrm{~b}$ & $0,34 \mathrm{~b}$ & $0,91 \mathrm{~b}$ & $1,25 \mathrm{c}$ & $0,74 \mathrm{c}$ & $1,99 \mathrm{c}$ \\
\hline $\mathrm{CV}(\%)$ & 23,2 & 30,4 & 20,8 & 28,3 & 18,9 & 14,6 \\
\hline
\end{tabular}

${ }^{(1)}$ Médias seguidas de mesma letra minúscula, nas colunas, não diferem entre si pelo teste Duncan a $5 \%$ de significância. ${ }^{(2)} \mathrm{DAE}=$ Dias após a emergência. ${ }^{(3)} \mathrm{CV}$ : Coeficiente de Variação. 
Para todos os experimentos os dados foram submetidos à análise de variância com teste $\mathrm{F}$, e as médias dos tratamentos agrupados pelo teste de Duncan a 1 e $5 \%$ de significância utilizando o programa estatístico Assistat.

Somente aos 25 DAE o tratamento com $T$. asperellum UFT 201 foi significativamente superior para todas as características avaliadas. Quanto à eficiência relativa (ER) aos $25 \mathrm{DAE}$, foi observado valor superior $(\mathrm{p}<0,01)$ para $\mathrm{o}$ tratamento com inoculação de T. asperellum UFT 201, com um aumento de $78 \%$ em relação à testemunha e $47 \%$ em relação ao tratamento com inoculação de T. harzianum (Figura 1).

Para o experimento com arroz, aos 20 e 45 DAE observou-se que o tratamento com $T$. asperellum UFT 201 foi superior $(\mathrm{p}<0,05)$ para a massa seca da parte aérea (MSPA), massa seca da raiz (MSR) e massa seca total (MST) em relação ao tratamento com inoculação de T. harzianum e a testemunha sem inoculação (Tabela 2). Quanto à eficiência relativa (ER) aos $45 \mathrm{DAE}$, foram observados valores superiores $(\mathrm{p}<0,01)$ para $\mathrm{O}$ tratamento com inoculação de T. asperellum UFT 201, com aumentos de $95 \%$ em relação à testemunha e $58 \%$ em relação ao tratamento com T. harzianum (Figura 1).

Quanto ao experimento com soja, nas avaliações aos 20 DAE, os tratamentos com as inoculações de $T$. asperellum UFT 201 e $T$. harzianum foram superiores $(\mathrm{p}<0,05)$ à testemunha sem inoculação, para as características avaliadas (Tabela 2). Aos 45 DAE, o tratamento com inoculação do T. asperellum UFT 201 foi superior $(\mathrm{p}<0,05)$ aos demais tratamentos. Quanto à ER aos 45 DAE, o tratamento inoculado com $T$. asperellum UFT 201 foi superior aos demais $(\mathrm{p}<0,01)$, com aumentos de 128 e $77 \%$ em relação aos tratamentos com testemunha e com inoculação de T. harzianum, respectivamente (Figura 1).

Para o feijão-caupi, aos 20 DAE, os valores foram superiores $(\mathrm{p}<0,05)$ para os tratamentos com inoculação de $T$. asperellum UFT 201 e com T. harzianum. Aos 45 DAP, o tratamento com inoculação do $T$. asperellum UFT 201 foi superior $(\mathrm{p}<0,05)$ aos demais tratamentos (Tabela 2). Quanto à eficiência relativa (ER) aos 45 DAE, o tratamento inoculado com $T$. asperellum UFT 201 foi superior aos demais $(\mathrm{p}<0,01)$, com aumentos de 84 e $69 \%$ em relação aos tratamentos testemunha e inoculação com T. harzianum, respectivamente (Figura 1). Os aumentos observados na massa seca da raiz (MSR em todas as espécies estudadas podem ter promovido o incremento da sanidade das plantas e da capacidade de absorção de água e nutrientes. Plantas que contenham esse micro-organismo associados as suas raízes ou na rizosfera tendem a ter melhor capacidade de sobrevivência e de absorver nutrientes em situações adversas e, consequentemente, tem vantagem produtiva em relação àquelas que não possuem Trichoderma em suas raízes (VERMA et al., 2007; MACHADO et al., 2012).

Diversas pesquisas têm sido realizadas com fungos do gênero Trichoderma spp., sendo que algumas linhagens desse fungo apresentam a capacidade para o controle biológico (SILVA et al., 2011; MACHADO et al., 2012). Considerando o biocontrole, são vários os mecanismos de ação utilizados por esses fungos, dentre os quais, destacam-se a produção de metabólitos e enzimas com propriedades antifúngicas, o hiperparasitismo e a competição por nutrientes (VERMA et al. 2007).
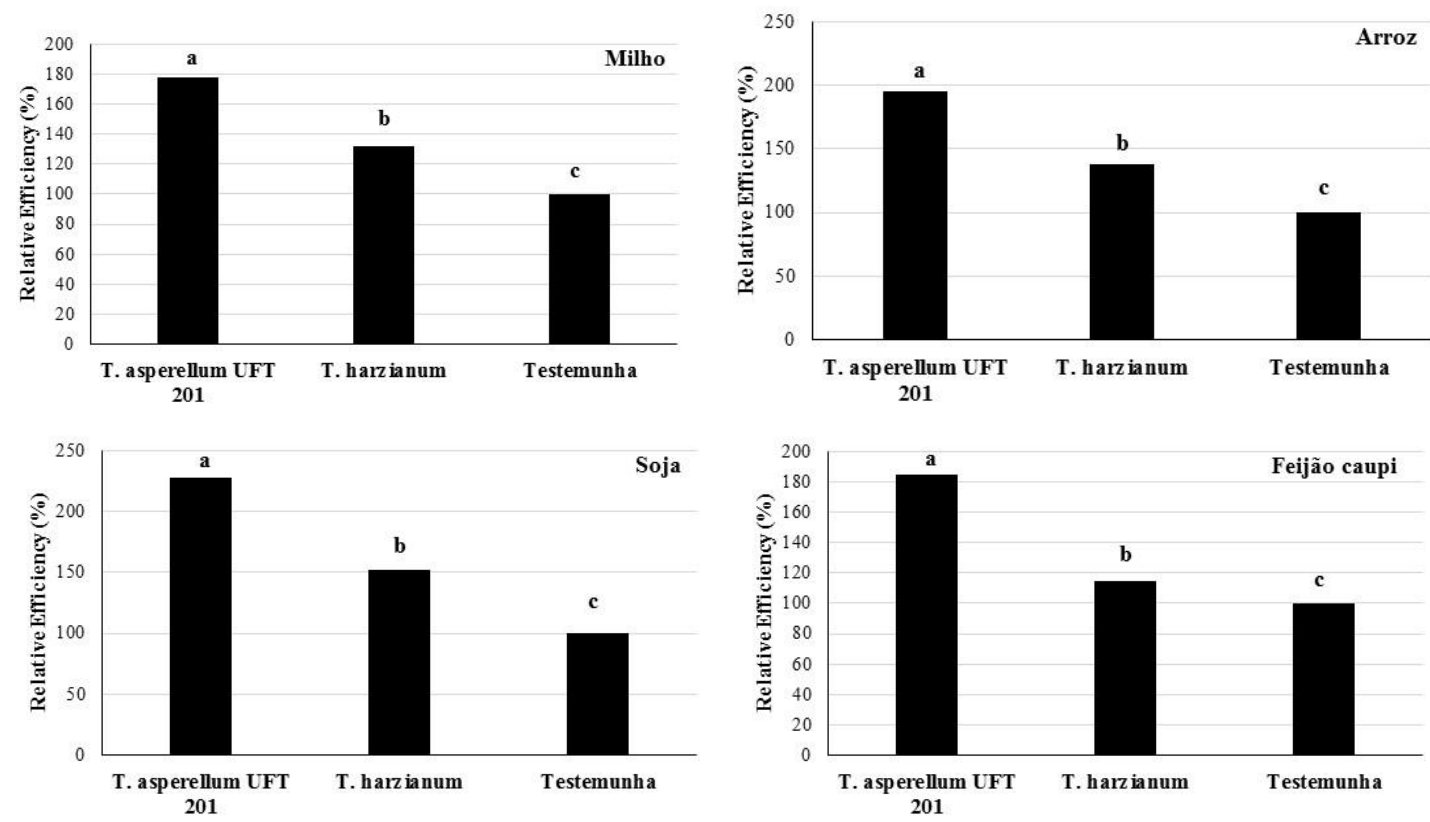

Figura 1. Eficiência relativa em milho, arroz, soja e feijão caupi, inoculadas com Trichoderma em casa de vegetação. Médias seguidas de mesma letra minúscula, não diferem entre si pelo teste Duncan a 5\% de significância. 
Além do controle biológico, alguns isolados de Trichoderma têm sido referidos como promotores do crescimento vegetal pela habilidade que possuem na solubilização de fosfatos e outros minerais (KAPRI; TEWARI, 2010; SILVA et al., 2011), disponibilizandoos para as plantas, bem como, pela produção de auxinas (VINALE et al., 2008; OLIVEIRA et al., 2012), que segundo Taiz e Zeiger (2006) são substâncias que apresentam propriedades de induzir a elongação celular nos vegetais superiores.

Segundo Baugh e Escobar (2007), a ação de Trichoderma como estimulador do crescimento é complexa e realizada por interações com fatores bioquímicos e produção de diversas enzimas e compostos benéficos para as plantas. Esta característica de promoção de crescimento pode ser observada na biomassa das espécies vegetais estudadas (Tabela 2) bem como na eficiência relativa que apresenta os ganhos em biomassa da parte aérea em relação ao tratamento testemunha sem inoculação (Figura 1). Corroboram com resultados reportados por Chagas et al. (2016) para o feijão caupi, Chagas et al. (2017) para arroz e Chagas et al. (2017) para soja onde houve o efeito positivo da inoculação de Trichoderma.

Para as gramíneas e as leguminosas, os resultados demonstram a capacidade do isolado de Trichoderma asperellum UFT 201 em promover o crescimento inicial de plantas, como observado para as culturas da soja, feijão caupi, milho e arroz. Estes resultados estão de acordo com outros trabalhos com diferentes culturas agrícolas inoculadas com diferentes isolados de Trichoderma, mesmo sendo com diferentes espécies de Trichoderma.

Santos et al. (2010) concluíram que o uso de Trichoderma spp. proporcionou resultados positivos no incremento de massa fresca e seca de plantas de maracujá oriundas de estacas. Carvalho et al. (2011) avaliaram a inoculação de isolados Trichoderma na promoção do crescimento inicial de feijoeiro comum. Jesus et al. (2011) ressaltaram o potencial de $T$. asperellum como condicionador de substrato para a produção de mudas de café, evidenciando o efeito positivo no aumento da biomassa da raiz, da parte aérea e total, bem como o aumento da eficiência da absorção de fósforo.

Silva et al. (2012) demostraram que isolados de Trichoderma obtidos de solos da Amazônia aumentaram a biomassa de plantas de arroz em casa de vegetação, mostrando também o potencial como promotores de crescimento. Machado et al. (2012) ressaltaram também, que pesquisas comprovam que Trichoderma spp. é eficiente, prático e seguro quanto aos métodos de aplicação, biocontrole e promoção de crescimento vegetal
O uso de Trichoderma asperellum UFT 201 apresenta resultados positivos no acúmulo de biomassa para as culturas da soja, feijão caupi, arroz e milho.

\section{Referências Bibliográficas}

ASUMING-BREMPONG, S. Phosphate solubilizing microorganisms and their ability to influence yield of rice. Agricultural Science Research Journal, Legon, v. 3, n. 12, p. 379-386, 2013.

BAUGH, C. L.; ESCOBAR, B. The genus Bacillus and genus Trichoderma for agricultural bio-augmentation. Rice Farm Magazine, Anytown, NY, v. 1, n. 4, p. 1-4, 2007

CARVALHO, D. D. C.; MELLO, S. C. M.; LOBO JUNIOR, M.; GERALDINE, A. M. Biocontrol of seed pathogens and growth promotion of common bean seedlings by Trichoderma harzianum. Pesquisa Agropecuária Brasileira, Brasília-DF, v. 46, n. 8 , p. $822-828,2011$.

CHAGAS, L. F. B.; CASTRO, H. G.; COLONIA, B. S. O.; CARVALHO FILHO, M. R.; MILLER, L. O.; CHAGAS JUNIOR, A. F. Efficiency of Trichoderma spp. as a growth promoter of cowpea (Vigna unguiculata) and analysis of phosphate solubilization and indole acetic acid synthesis. Brazilian Journal of Botany, São Paulo-SP, v. 38, n. 4, p. 111, 2016.

CHAGAS, L. F. B.; COLONIA, B. S. O.; SANTOS, G. R.; SCHEIDT, G. N.; PORTELLA A. C. F.; SOARES, L. P.; CHAGAS JUNIOR, A. F. Rice growth influence by Trichoderma spp. with natural phosphate fertilization under greenhouse conditions. International Journal of Development Research, v. 07, n. 6, p. 13147-13152, 2017.

CHAGAS, L. F. B.; CHAGAS JUNIOR, A. F.; FIDELIS, R. R.; CARVALHO FILHO, M. R.; MILLER, L. O. Trichoderma asperellum efficiency in soybean yield components. Comunicata Scientiae, Teresina-PI, v. 08, n. 1, p. 165-169, 2017.

CONTRERAS-CORNEJO, H. A.; MACÍAS-RODRÍGUES, L.; CORTÉS-PENAGOS, C.; LÓPEZ-BUCIO, J. Trichoderma virens, a plant beneficial fungus, enhances biomass production and promotes lateral root growth through an auxin-dependent mechanism in Arabidopsis. Plant Physiology, United States, v. 149, n. 3, p. 1579-1592, 2009.

EMBRAPA. EMPRESA BRASILEIRA DE PESQUISA AGROPECUÁRIA. Manual de análises químicas de solos, plantas e fertilizantes. 2 ed. Brasília-DF, 2009. 627 p.

HOYOS-CARVAJAL, L.; ORDUZ, S.; BISSETT, J. Genetic and metabolic biodiversity of Trichoderma from Colombia and adjacent neotropic regions. Fungal Genetics and Biology, Madison, v. 46, n. 9, p. 615-631, 2009.

JESUS, E. P.; SOUZA, C. H. E.; POMELLA, A. W. V.; COSTA, R. L.; SEIXAS, L.; SILVA, R. B. Avaliação do potencial de Trichoderma asperellum como condicionador de substrato para a produção de mudas de café. Cerrado Agrociência, Patos de Minas-MG, v. 2, n.2, p. 7-19, 2011.

KAPRI, A.; TEWARI, L. Phosphate solubilization potential and phosphatase activity of rhizospheric Trichoderma spp. 
Brazilian Journal of Microbiology, São Paulo-SP, v. 41, n. 3, p. 787-795, 2010.

MACHADO; D. F. M.; PARZIANELLO, F. R.; SILVA, A. C. F.; ANTONIOLLI, Z. I. Trichoderma no Brasil: O fungo e o bioagente. Revista de Ciências Agrárias, Lisboa, v. 35, n. 1, p. 274-288, 2012.

OLIVEIRA, A. G.; CHAGAS JÚNIOR, A. F.; SANTOS, G. R.; MILLER, L. O.; CHAGAS, L. F. B. Potencial de solubilização de fosfato e produção de AIA por Trichoderma spp. Revista Verde de Agroecologia e Desenvolvimento Sustentável, Pombal, v. 7, n. 3, p. 149-155, 2012.

POMELLA; A. W. V.; RIBEIRO, R. T. S. Controle biológico com Trichoderma em grandes culturas - uma visão empresarial. In: BETTIOL, W.; MORANDI, M. A. B. (Eds.). Biocontrole de doenças de plantas: uso e perspectivas. Jaguariúna: Embrapa Meio Ambiente, 2009. p. 239-244.

SAMUELS, G. J.; ISMAIEL, A.; BON, M. C.; RESPINIS, S.; PETRINI, O. Trichoderma asperellum sensu lato consists of two cryptic species. Mycologia, Lawrence, v. 102, n. 4, p. 944-966, 2010.

SANTOS, C. C.; OLIVEIRA, F. A.; SANTOS, M. S.; TALAMINI, V.; FERREIRA, J. M. S.; SANTOS, F. J. dos. Influência de Trichoderma spp. sobre o crescimento micelial de Thielaviopsis paradoxa. Scientia Plena, Maceió-AL, v. 8, n. 4. p. 1-5, 2012.
SANTOS, H. A.; MELlO, S. C. M.; PEIXOTO, J. R. Associação de isolados de Trichoderma spp. e ácido indol-3butírico (AIB) na promoção de enraizamento de estacas e crescimento de maracujazeiro. Bioscience Journal, Uberlândia-MG, v. 26, n. 6, p. 966-972, 2010.

SILVA, J. C.; TORRES, D. B.; LUSTOSA, D. C.; FILIPPI, M. C. C.; SILVA, G. B. Rice sheath blight biocontrol and growth promotion by Trichoderma isolates from the Amazon. Amazonian Journal of Agricultural and Environmental Sciences, Belém-PA, v. 55, n. 4, p. 243-250, 2012.

SILVA, V. N.; GUZZO, S. D.; LUCON, C. M. M.; HARAKAVA, R. Promoção de crescimento e indução de resistência à antracnose por Trichoderma spp. em pepineiro. Pesquisa Agropecuária Brasileira, Brasília-DF, v. 46, n. 12, p. 1609-1618, 2011.

TAIZ, L.; ZEIGER, E. Plant Physiology. 4 ed. Sunderland: Sinauer Associates, Inc. Publishers, 2006. 819 p.

VERMA, M.; BRAR, S. K.; TYAGI, R. D.; SURAMPALLI, R. Y.; VALÉRO, J. R. Antagonistic fungi, Trichoderma spp.: Panoply of biological control. Biochemical Engineering Journal, Amsterdam, v. 37, n. 1, p. 1-20, 2007.

VINALE, F.; SIVASITHAMPARAM, K.; GHISALBERT, E. L.; MARA, R.; BARBATTI, M. J.; LI, H.; WOO, S. L.; LORITO, M. A novel role for Trichoderma secondary metabolites in the interactions with plants. Physiological and Molecular Plant Pathology, Amsterdam, v. 72, n.3, p. 80-86, 2008 